\title{
Study of the operational SNR while constructing polar codes
}

\author{
Reda Benkhouya ${ }^{1}$, Idriss Chana ${ }^{2}$, Youssef Hadi $^{3}$ \\ ${ }^{1,3}$ MISC, Faculty of Sciences, Ibn Tofail University, Morocco \\ ${ }^{2}$ ISIC EST-LMMI ENSAM, Moulay Ismail University, Morocco
}

\begin{tabular}{l}
\hline \hline Article Info \\
\hline Article history: \\
Received May 30, 2019 \\
Revised Dec 14, 2019 \\
Accepted Jan 8, 2020 \\
\hline
\end{tabular}

Keywords:

Bhattacharyya parameter

Polar codes

SNR

Successive cancellation decoding

\begin{abstract}
Channel coding is commonly based on protecting information to be communicated across an unreliable medium, by adding patterns of redundancy into the transmission path. Also referred to as forward error control coding (FECC), the technique is widely used to enable correcting or at least detecting bit errors in digital communication systems. In this paper we study an original FECC known as polar coding which has proven to meet the typical use cases of the next generation mobile standard. This work is motivated by the suitability of polar codes for the new coming wireless era. Hence, we investigate the performance of polar codes in terms of bit error rate (BER) for several codeword lengths and code rates. We first perform a discrete search to find the best operational signal-to-noise ratio (SNR) at two different code rates, while varying the blocklength. We find in our extensive simulations that the BER becomes more sensitive to operational SNR (OSNR) as long as we increase the blocklength and code rate. Finally, we note that increasing blocklength achieves an SNR gain, while increasing code rate changes the OSNR domain. This trade-off sorted out must be taken into consideration while designing polar codes for high-throughput application.
\end{abstract}

Copyright $(0) 2020$ Institute of Advanced Engineering and Science. All rights reserved.

\section{Corresponding Author:}

Reda Benkhouya,

MISC, Faculty of Sciences,

Ibn Tofail University,

Av. de L'Université, Kénitra, Morocco.

Email: reda.benkhouya@gmail.com

\section{INTRODUCTION}

Over last years, wireless applications have been growing fast and requiring more challenging coding schemes. Recently, polar codes were proven to be capacity achieving for binary discrete memoryless channel (B-DMC). Combined with high order modulation, polar code is powerful candidate for the next generation mobile standard, where high transmission power efficiency and bandwidth efficiency are required [1]. Fortunately, both polar encoding and decoding are of low complexity, so need minimum time and space, and this match green energy requirement [2]. The performance of polar codes enables their application in varying channels, which tackles the universal coverage requirement. On the other side, complex use scenarios result in heterogeneous networks where polar codes can find their suitable applications. Therefore, these codes have become one popular topic and drawn intensive attentions from both academia and industry. Recent research progresses on polar codes can be mainly categorized into two trends: 1) advanced decoding algorithms for polar codes, and 2) efficient hardware implementation methods for polar codes [2].

Polar codes are linear block codes that rely on a polarization phenomenon. The advent of polar codes is based on the channel polarization theory. The core idea of polar coding is to split a given vector channel into multiple correlated bit channels and to use only the good ones, in the sense that they are either extremely noisy or noiseless. Then, one can employ a separate sequential decoder on each sub-channel. Polar codes have performed state-of-the-art codes of larger block lengths and code rates. Polar codes can also 
be used for source coding, but this use will not be discussed in this paper. In the level of theoretical analysis and development, substantial recent research progress has been achieved and reported. A by no means complete list of references is [3, 4], see also [5] and the references therein. The aim of this paper is to demonstrate how the code changes with the OSNR and the block length as well as the code rate for the additive white Gaussian noise (AWGN) channel. Since it is not known what impact has the OSNR on the BER performance of polar codes, this paper aims to open the related discussion. As concluded in [6] all polar code constructions are equally good in AWGN if the OSNR is optimized for the best performance. Thus in our work we may use simple algorithm only, namely Bhattacharyya bounds based construction. The readers wanting to go deeper into the current study may experiment separately the orthogonal frequencydivision multiplexing (OFDM) [7-24] and exploit our approach to get the effect of such modulation technique on the performance of the code construction. Obviously, OFDM is still an ultimate candidate for wireless applications, since it allows resolving the well-known problem of spectrum underutilization [25]. The remainder of this paper is outlined as follows. Section 2 describes polar codes that we study in this work. In Section 3 we review a simplest construction to select the bit-channels over which the information bits are transmitted. The decoder adopted is introduced in Section 4. Afterwards, simulation results are provided in Section 5. Finally, conclusions and future works follow in Section 6.

\section{POLAR CODING}

Let $(N, K)$ be a linear bock code, where $N=2^{n}$ is the code length, $n$ is an arbitrary integer, $K$ is the code dimension, the code rate of such coding scheme is defined by $R=K / N, 0 \leq K \leq N$. A binary polar code is completely specified by a triple $(N, K, \mathfrak{F})$, where $\mathfrak{F} \subseteq \mathbb{N},|\mathfrak{F}|=N-K$ is the set of the frozen bit indices. The remaining $K$ elements are called the information bits indices. Let $F^{\otimes n}=F \otimes \ldots \otimes F$ be the n-fold Kronecker product of Arikan's standard polarizing kernel $F \triangleq\left[\begin{array}{ll}1 & 1 \\ 0 & 1\end{array}\right]$. The matrix $F^{\otimes n}$ denotes the n-th tensor power of $F$ and could be evaluated by applying the Kronecker product recursively according to $F^{\otimes n}=F^{\otimes(n-1)} \otimes F$. Then for a vector of information bits $u$ of length $K$, a codeword is generated as

$$
x=G \cdot u
$$

where $G \triangleq\left(F^{\otimes n}\right)_{\mathbb{F}^{c}}$ is the generator matrix of polar code which picks a specific subset of $K$ rows of the $N \times N$ matrix, and $\mathfrak{F}^{C} \triangleq\{0,1, \ldots, N-1\} \backslash \mathfrak{F}$ corresponds to the set of non-frozen bit indices. Implicitly, with respect to classical $N \times N$ matrix based encoding, the frozen bits $\mathfrak{F}$ are set to zero and we follow this convention throughout the paper. Consider a channel $\mathrm{W}$ is used for transmitting the information between input and output. Let $x=\left(x_{1}, x_{2}, \ldots, x_{N}\right)$ be the inputs vector and $y=\left(y_{1}, \ldots, y_{N}\right)$ be the outputs vector. Given a binary-input channel $W: X \rightarrow Y$ with $X=\{0,1\}$, the Bhattacharyya parameter $Z(W)$ can be used to measure the error performance of the channel. In general, we can choose the positions of the information bits and frozen bits by their Bhattacharyya parameter $Z(W)$, which can be defined as the upper bound of the decision error probability when the channel is used to transmit zero or one as follows:

$$
Z(W) \triangleq \sum_{y \in Y} \sqrt{p(y \mid 0) p(y \mid 1)}
$$

where $p(y \mid s)$ is the conditional probability of the received $y$ provided that $s \in\{0,1\}$ is transmitted. As the bit-channels start polarizing, they approach either noiseless good bit-channel or a pure-noise bad bitchannel. The Bhattacharyya parameter indicates that the fraction of bit channels approaches the mutual information $I(W)$ as $n \rightarrow \infty$. Even though systematic variants of polar encoding do exist, we construct the original polar codes which are non-systematic, and being a linear code, the encoding simply needs a matrix multiplication. Once the code size is larger, matrix multiplication becomes computationally expensive as far as $O\left(N^{2}\right)$. Thus, in our work we use an alternative implementation based on FFT's butterfly circuit model, which exhibits significantly reduced computational complexity of $O(N \log N)$. In our polar code design, we use recursive estimation of the just introduced Bhattacharyya parameters of bit-channels, which is going to be detailed in the next section. 


\section{THE CODE CONSTRUCTION}

Recall that polar code construction is ranking algorithm that selects $K$ best among $N$ possible polar bit-channels, in terms of the bit error rate at a given initial value defined as the OSNR. The choice of the set of frozen bit $\mathfrak{F}$ is an important step in polar coding often referred to as polar code construction. The original algorithm of polar codes is based on the evolution of simple bounds on the Bhattacharyya parameters of bit channels. Due to its simplicity, this construction has been widely used, and produced good polar codes. The basic idea is to create a coding system where one can access each bit-channel individually and send data only through those for its Bhattacharyya parameter is close to 0 . The Bhattacharyya parameter $Z(W)$ is an upper bound on the error probability of transmission over $W$ with maximum likelihood (ML) decision when the channel is used only once to transmit a 0 and 1. Intuitively, channels with $Z(W) \leq \varepsilon$ are almost noiseless, while channel with $Z(W) \geq 1-\varepsilon$ are almost pure-noise channel for a given $0 \leq \varepsilon \leq 1$. The Bhattacharyya parameter of channel plays an important part in the construction of Polar codes. For a more detailed exposition, we confer the following recursion for $j=0, \ldots, n-1$ with initial $z_{0,0}=e^{-R S}$ chosen to optimize the code performance at a certain ( $R S$ ), where $S$ is the SNR value.

$$
z_{j+1, i}=\left\{\begin{array}{lc}
2 z_{j, i}-z_{j, i}^{2} & 0 \leq i \leq 2^{j} \\
z_{j, i-2^{j}}{ }^{2} & 2^{j} \leq i \leq 2^{j+1}
\end{array}\right.
$$

The indices of the highest $N-K$ values in the set of $N$ final stage values $\left\{z_{n, i}: i=0, \ldots, N-1\right\}$ form the set $\mathfrak{F}$. The code rate $R$ can be varied by adding or deleting subchannels from the good subchannel set. With code length $N$ increasing to infinity, bit-channels polarize to be nearly noiseless or useless [1]. After performing channel polarization transform, the good subchannels are assigned information bits and the bad ones are set frozen bits. Let such a channel be defined by the transition probabilities $W(y \mid x), x \in X=\{0,1\}$ and $y \in Y$. Based on (2), the definition of the Bhattacharyya parameter of $W$ extended from discrete to continuous channel is given by

$$
Z(W)=\int W(y \mid 0) W(y \mid 1) d y
$$

where $W(y \mid s)$ is the transition probability of receiving $y$ when $s \in\{0,1\}$ has been sent. Then we analyze the initial value of $Z(W)$ for Gaussian channel. At first, the initial value of Bhattacharyya parameter is definitely difficult since channels are continuous. Suppose there is a communication link with Gaussian noise with expectation zero and variance $\sigma^{2}$. At the same time, BPSK is used as modulation. The recursive algorithm of construction requires an initial value, corresponding to the worst BER and may be replaced with $e^{-R E_{b} / N_{0}}$, where $E_{b}$ the energy spent per each information bit and $N_{0} / 2=\sigma^{2}$. Moreover, the initial value of Bhattacharyya parameter is not suitable for all communication channels since polar codes are channel specific designs. By definition of polar codes, the construction should be repeated at every time channel changes. Nevertheless, we wish to construct a polar code at one OSNR and use it for a range of possible SNRs. This way, we use a unique code created by running the code-construction at a single value of the channel-state and keeping the code unchanged for all channel conditions. As we see later, it is crucial to properly select the adequate OSNR given rate and blocklength for the performance in terms of bit error rate.

\section{THE NATIVE DECODING}

It is proven in [26] that for any $(N, K)$ polar code on any B-DMC, there exists an encoder and a decoder known as successive cancellation (SC) decoder, each with the same order of complexity $O(N \log N)$. We assume that the decoder considered in the system is a SC decoder, for which polar codes are tailored. SC algorithm, which decodes each bit in a successive manner, is usually employed and proposed as sub-optimal approach. By taking advantage of the polarization effect, polar codes can achieve the symmetric capacity of binary memoryless channels with low complexity SC decoding strategy [1]. Being fundamental for all the later advanced decoders that exhibit superior performance, one cannot avoid 
having an SC decoder. The SC algorithm traverses the entire polar code tree depth first, visiting all leaf nodes [27]. From decoding viewpoint, constructing a polar code of dimension $K$ is equivalent to finding the $K$ best bit-channels that model the channel that the decoder sees when it recovers one by one the information bits corresponding to the received codeword by the SC decoder [28]. According to the construction of polar codes, two groups of bits are established from the many independent copies of channels. The first group is the information bits $U_{\mathfrak{F}^{c}}=\left(u_{i}: 1 \leq i \leq K\right)$ and the second one is the frozen bits $U_{\mathfrak{F}}=\left(u_{j}: 1 \leq j \leq N-K\right)$ that are made known to the decoder. The log-likelihood ratios (LLR) of the channel are calculated as

$$
\operatorname{LLR}\left(y_{i}\right)=\ln \frac{p\left(y_{i} \mid x_{i}=0\right)}{p\left(y_{i} \mid x_{i}=1\right)}
$$

The SC applies the recursive calculations on the received LLRs from (5) and the decision function for the SC decoder is defined by

$$
\hat{U}_{i}= \begin{cases}0, & \operatorname{LLR}\left(y_{1}{ }^{N}, U_{1}^{i-1}\right) \geq 0 \\ 1, & \operatorname{LLR}\left(y_{1}{ }^{N}, U_{1}^{i-1}\right)<0\end{cases}
$$

where $\operatorname{LLR}\left(y_{1}{ }^{N}, U_{1}^{i-1}\right)$ is equivalent to the likelihood ratio of $U_{i}$ given the channel output $Y$ and $U_{1}^{i-1}$ which are found previously by the decoder. It should be mentioned that, for the first bit $\hat{U}_{1}$ value, the decoder uses only $y$ for the decision. In our simulation we use an implementation of the basic successive cancellation decoder, which we believe to be the simplest implementation possible.

\section{NUMERCIAL RESULTS AND DISCUSSION}

In this section we report the results of our simulations. We consider comparing the performance of polar codes from various parameters. Even if the error performance of polar codes with short codeword length is already described by [4] as mediocre under SC algorithm, we insist on considering blocklength $N=256$ in addition to two new blocklengths, namely $N=1024, N=4096$. For each blocklength we consider only high code rate $R=5 / 6$. The conditions of simulation are described in Table 1 .

Table 1. Simulation parameters

\begin{tabular}{ll}
\hline Parameter name & Value \\
\hline Minimum block samples & 1000 \\
Minimum bit errors & 100 \\
Modulation & BPSK \\
Channel & AWGN \\
\hline
\end{tabular}

The transmission is supposed to be over an AWGN channel with zero mean. Without loss of generality, we normalize the noise variance to be unity in the remainder of our simulation. Bits in codeword are modulated using binary phase shift keying (BPSK). We ensure a practical values of BER (e.g. order of $10^{-3}$ and less), and guarantee a minimum of 1000 block samples. For each evaluated SNR, we achieve at least 100 bit errors. In order to study the impact of OSNR on BER performance, we use the discrete search of OSNR over a finite interval. We carry out simulations and we plot the results in logarithmic domain. The discrete search consists on spanning the whole interval starting from the OSNR -5dB as initial point when necessary. The search is pursued further by incrementing OSNR as long as the BER improves. We stop the discrete search once the BER performance degrades for at least one from the next OSNRs. By this way, we retrieve the candidate OSNR whose BER curve is the first one, which crosses the uncoded curve. We confirm the BER degradation by using some greater value of OSNR (e.g. 10dB). Note that in each figure, the important portion of the BER curves is magnified, where curves of studied codes cross the curve of the uncoded system.

Figure 1 illustrates SNR versus BER for $N=256$ at $R=5 / 6$. It can be noted that almost all OSNRs offer the same performance. It is also shown that the uncoded case offers better performance in terms of BER than the coded one since SNR is less than $3.77 \mathrm{~dB}$. According to our adopted method based on discrete search, the candidate OSNR is $1 \mathrm{~dB}$. Increasing $N$ to 1024 makes the performance weakly sensitive to 
the OSNR, as depicted in Figure 2. It is shown once again that the uncoded case offers better performance than the coded one since SNR is less than $3.65 \mathrm{~dB}$ for $R=5 / 6$. From the zoom part, the candidate OSNR is $3 \mathrm{~dB}$ for $R=5 / 6$.

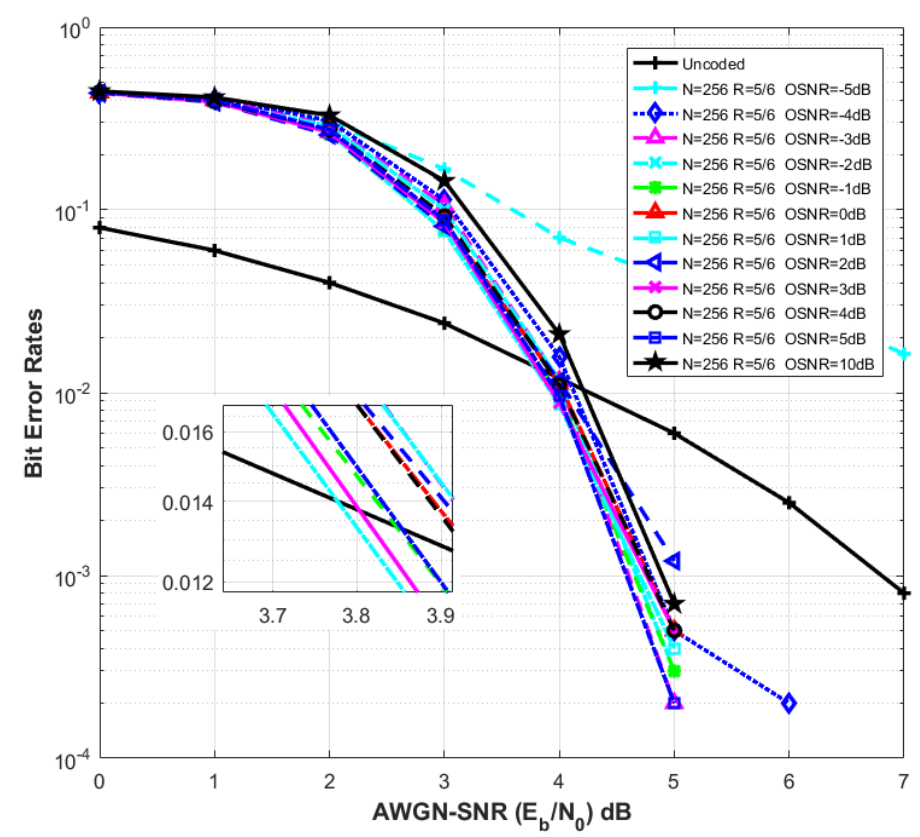

Figure 1. Impact of OSNR on BER performance for $N=256$ at $R=5 / 6$

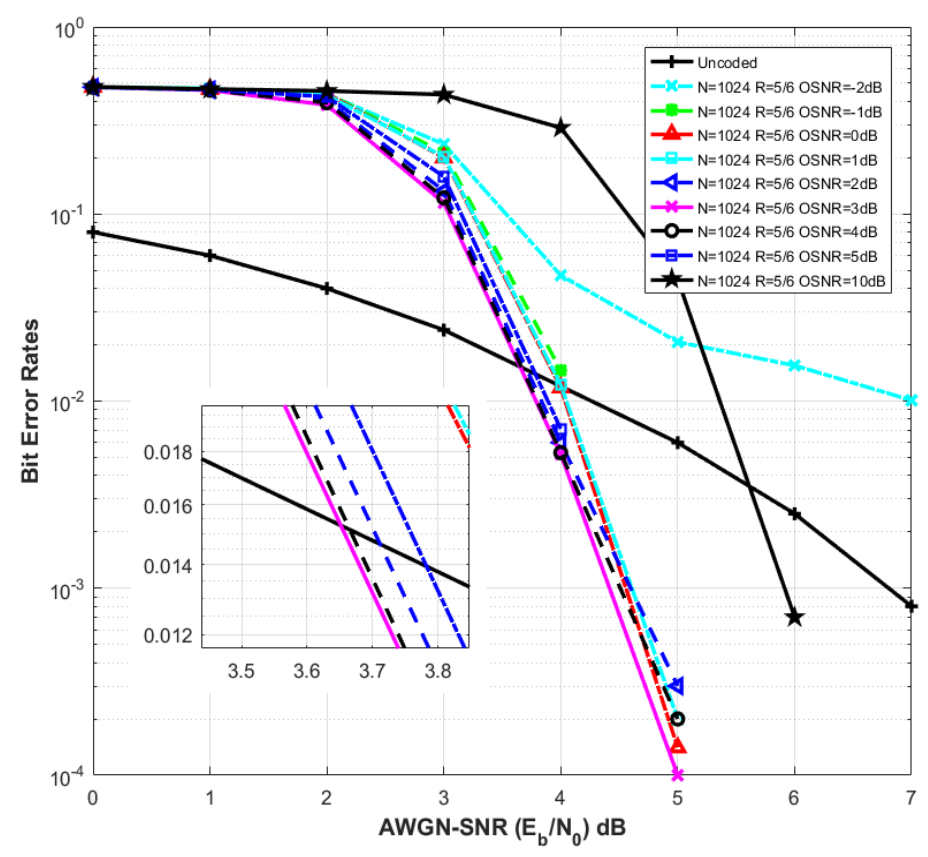

Figure 2. BER sensitivity to OSNR for $N=1024$ at $R=5 / 6$

Figure 3 depicts the performance of polar codes produced for $N=4096$ at $R=5 / 6$. Unlike smaller blocklength, it is shown that for $N=4096$ the BER becomes plainly sensitive to OSNR. The uncoded system offers better performance than the coded one in terms of BER since SNR is less than 3.468dB. It can be noted that the candidate OSNR is $5 \mathrm{~dB}$. For instance, the BER degradation starts at OSNR > 5dB and degrades dramatically when OSNR reaches $10 \mathrm{~dB}$. As recapitulation, increasing blocklength makes BER performance 
sensitive to OSNR. It is indeed critical to construct polar code with a good BER performance. This has been already stated in [6] but only for blocklength $N=2048$ and code rate $R=1 / 2$. To avoid confusion and stress the differences, let us summarize the results in Table 2. The candidate OSNR corresponds to a selected value according to the discrete search method. Figure 4 illustrates the construction chart for code rate $R=5 / 6$. Clearly for a given code rate $R$; the higher blocklength is, the earlier the coded system outperforms the uncoded one.

The curves demonstrate the BER performance versus SNR for the code rate $R=5 / 6$. While constructing polar code with rate $R=5 / 6$, increasing blocklength from $N=256$ to $N=4096$ achieves the SNR gain of $\Delta \mathrm{Gain}=1.2 \mathrm{~dB}$ at the BER of $10^{-4}$. This trade-off sorted out must be taken into consideration while constructing polar codes for high-throughput application.

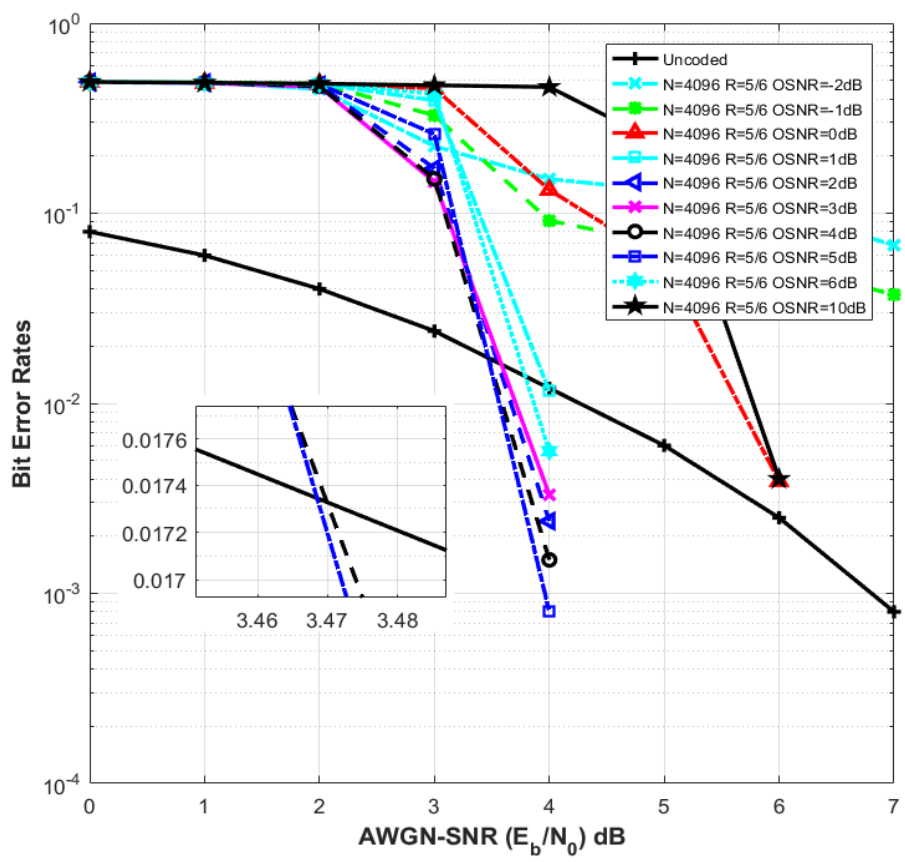

Figure 3. Impact of OSNR on BER performance for $N=4096$ at $R=5 / 6$

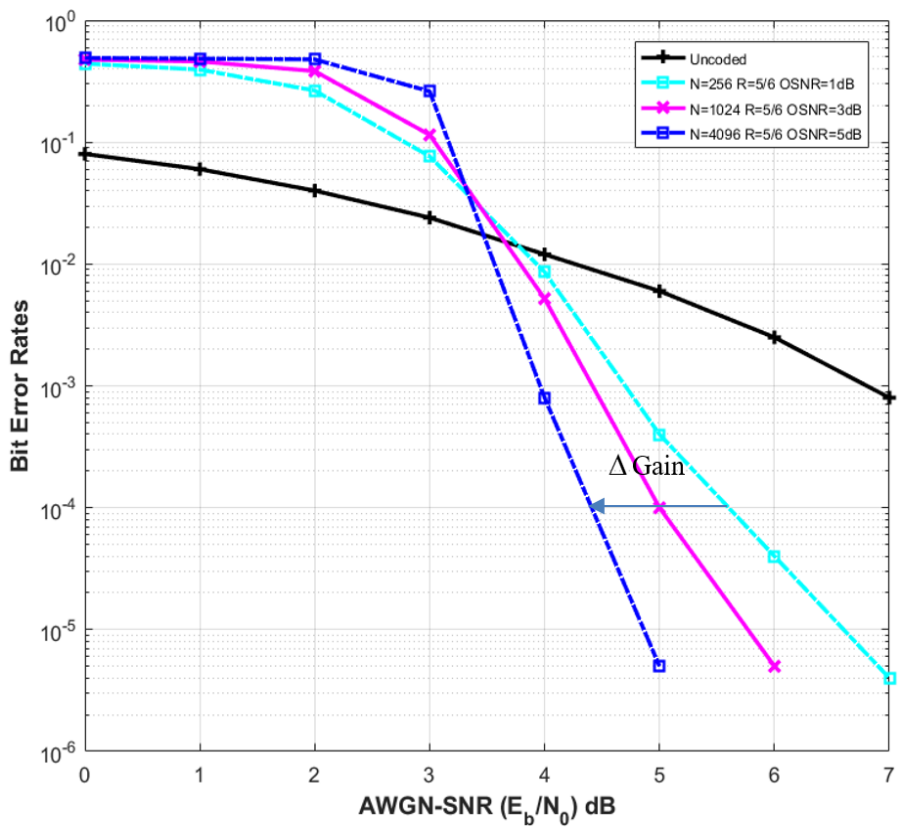

Figure 4. Construction chart for code rate $R=5 / 6$ 
Table 2. Impact of OSNR on BER sensitivity for code rate $R=5 / 6$

\begin{tabular}{ccc}
\hline Code length & Sensibility & Candidate OSNR \\
\hline 256 & Low & 1 \\
1024 & Medium & 3 \\
4096 & High & 5 \\
\hline
\end{tabular}

\section{CONCLUSION}

We restudied in this paper the non-universality of polar codes. We performed a discrete search to find the best OSNR for code construction. We then compared BER performances for three codeword lengths and observed that for short one the effect of OSNR is meaningless even for higher code rate. It is also observed that for long blocklength, OSNR have huge impact on BER performance. In this paper, the BER performance analysis is summarized to clarify discussion on construction perspective. We assume that the proposed chart contributes to pave the path towards this end. The results shown are preliminary and the work needs to be extended further to prove that the study is indeed viable for Rayleigh fading channel, or even Nakagami-m fading channel. We intend to investigate in a future work the performance analysis of the considered construction under orthogonal frequency-division multiplexing (OFDM). Such system authorizes division of the bandwidth into narrow orthogonal sub channel, and this is useful to optimize the spectrum use. However, concerning polar codes, the decoding performance of SC algorithm is still not satisfying. We may obtain better performance using an iterative decoder. There is also a need to study the effect of initial value of Bhattacharyya parameter on system's performance in a future work.

\section{REFERENCES}

[1] P. Chen, M. Xu, B. Bai and X. Ma, "Design of polar coded 64-QAM," 2016 9th International Symposium on Turbo Codes and Iterative Information Processing (ISTC), Brest, pp. 251-255, 2016.

[2] L. Liu and C. Zhang, "Circuits and systems for 5G network: Massive MIMO and advanced coding," 2015 IEEE 11th International Conference on ASIC (ASICON), Chengdu, pp. 1-4, 2015.

[3] T. C. Gulcu, M. Ye and A. Barg, "Construction of polar codes for arbitrary discrete memoryless channels," 2016 IEEE International Symposium on Information Theory (ISIT), Barcelona, pp. 51-55, 2016.

[4] T. Wang, D. Qu and T. Jiang, "Parity-Check-Concatenated Polar Codes," in IEEE Communications Letters, vol. 20, no. 12 , pp. $2342-2345$, Dec. 2016.

[5] E. Arikan, "On the Origin of Polar Coding," IEEE Journal on Selected Areas in Communications, vol. 34, no. 2, pp. 209-223, 2016.

[6] H. Vangala, E. Viterbo and Y. Hong, "A comparative study of Polar Code Constructions for the AWGN Channel," pp. 1-9, 2015.

[7] G. A. Hussain, L. Audah, "BCH codes for 5G wireless communication systems over multipath fading channel," Indonesian Journal of Electrical Engineering and Computer Science (IJEECS), vol. 17, no. 1, pp. 310-316, 2020.

[8] P. Manhas, M.K Soni, "OFDM System Performance Evaluation under Different Fading Channels and Channel Coding using Matlab Simulink," Indonesian Journal of Electrical Engineering and Computer Science (IJEECS), vol. 5, no. 2, pp. 260-266, 2017

[9] P. S. Swapna, S. Pillai Sakuntala, "Resource Allocation Algorithm for Symmetrical Services in OFDMA Systems," Indonesian Journal of Electrical Engineering and Computer Science (IJEECS), vol. 18, no. 2, pp. 867-874, 2020.

[10] J. Shukla, A. Joshi, and R. Tyagi, "PAPR analysis of OFDM system using AI based multiple signal representation methods," TELKOMNIKA (Telecommunication, Computing, Electronics and Control), vol. 17, no. 2, pp. 2983-2991, 2020.

[11] M. K. Husein, "Minimize MIMO OFDM interference and noise ratio using polynomial-time algorithm," International Journal of Electrical and Computer Engineering (IJECE), vol. 10, no. 1, pp. 900-907, 2020.

[12] B. Sabbar, M. Khalifa, "Performance Evaluation of High Mobility OFDM Channel Estimation Techniques," International Journal of Electrical and Computer Engineering (IJECE), vol. 10, no. 3, pp. 2562-2568, 2020.

[13] S. Razmi, N. Parhizgar, "Adaptive resources assignment in OFDM-based cognitive radio systems," International Journal of Electrical and Computer Engineering (IJECE), vol. 9, no. 3, pp. 1935-1943, 2019.

[14] S. Suyoto, I. Iskandar, and S. Sugihartono, Adit Kurniawan, "Improved Timing Estimation Using Iterative Normalization Technique for OFDM Systems," International Journal of Electrical and Computer Engineering (IJECE), vol. 7, no. 2, pp. 905-911, 2017.

[15] H. Hamlili, S. Kameche, and A. Abdelmalek, "S $\alpha$ S noise suppression for OFDM wireless communication in rayleight channel," International Journal of Electrical and Computer Engineering (IJECE), vol. 10, no. 2, pp. 2003-2010, 2020.

[16] B. Archana B., and T. P. Surekha, "Integrated approach for efficient power consumption and resource allocation in MIMO-OFDMA," International Journal of Electrical and Computer Engineering (IJECE), vol. 10, no. 2, pp. 2069-2076, 2020.

[17] A.H. Ali, and A. A. Hussien, "Comprehensive Investigation of Coherent Optical OFDM-RoF Employing 16QAM External Modulation for Long-Haul Optical Communication System," International Journal of Electrical and Computer Engineering (IJECE), vol. 10, no. 3, pp. 2607-2616, 2020. 
[18] M.I. Youssif, A.E. Emam, M.A. ElGhany, "Image multiplexing using residue number system coding over MIMOOFDM communication system," International Journal of Electrical and Computer Engineering (IJECE), vol. 9, no. 6, pp. 4815-4825, 2019.

[19] M. D. Rozaini, A. Idris, D. M. Ali, E. Abdullah, "Median codeword Shift (MCS) technique for PAPR reduction with low complexity in OFDM system," International Journal of Electrical and Computer Engineering (IJECE), vol. 9 , no. 6, pp. 4882-4888, 2019

[20] M. F. Ghanim, "Performance investigation of WOFDM for 5G wireless networks," International Journal of Electrical and Computer Engineering (IJECE), vol. 9, no. 4, pp. 3153-3158, 2019

[21] K. J. Thajeel, I. K. Abboud, L. A. Kunbar, "Design of encrypted transceiver Non-coherent OFDM with ability to correct coding bits of information," International Journal of Electrical and Computer Engineering (IJECE), vol. 9, no. 4, pp. 3212-3220, 2019.

[22] A. H. Ali, H. J. Alhamdane, Begared Salih Hassen, "Design analysis and performance evaluation of the WDM integration with CO-OFDM system for radio over fiber System," Indonesian Journal of Electrical Engineering and Computer Science (IJEECS), vol. 15, no. 2, pp. 870-878, 2019.

[23] S. Atoui, N. Doghmane, S. Afifi, "Blind frequency offset estimator for OFDM systems," TELKOMNIKA (Telecommunication, Computing, Electronics and Control), vol. 17, no. 6, pp. 2722-2728, 2019.

[24] Rawaa J. Hasan, and Hikmat N. Abdullah, "Comparative study of selected subcarrier index modulation OFDM schemes," TELKOMNIKA (Telecommunication, Computing, Electronics and Control), vol. 17, no. 1, pp. 15-22, 2019.

[25] Z. M Abid, A. A Jaffaar, S. Q Hadi, "PAPR reduction in OFDM system for DVB-S2," Indonesian Journal of Electrical Engineering and Computer Science (IJEECS), vol. 17, no. 1, pp. 317-323, 2020

[26] A. Hadi, K. M. Rabie and E. Alsusa, "Polar codes based OFDM-PLC systems in the presence of middleton class-A noise," 2016 10th International Symposium on Communication Systems, Networks and Digital Signal Processing (CSNDSP), Prague, pp. 1-6, 2016.

[27] P. Giard et al., "Hardware decoders for polar codes: An overview," 2016 IEEE International Symposium on Circuits and Systems (ISCAS), Montreal, QC, pp. 149-152, 2016.

[28] M. Bardet, V. Dragoi, A. Otmani and J. Tillich, "Algebraic properties of polar codes from anew polynomial formalism," IEEE International Symposium on Information Theory, pp. 230-234, 2016.

\section{BIOGRAPHIES OF AUTHORS}

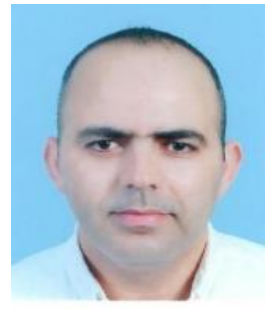

Reda Benkhouya was invited as a visiting scholar at Toulouse institute of computer science research (IRIT), France, in 2004. Since 2006 he has been with Etisalat where he is currently an ITSM. He is now pursuing the Ph.D. degree at Ibn Tofail University. His research interest includes channel coding, cognitive radio, artificial intelligence, IoT, cloud and big data.

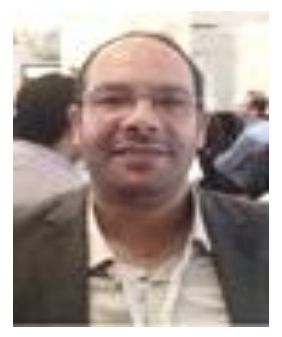

Idriss Chana received the Ph.D. degree from Mohamed V University, Morocco in 2013. He is currently a full professor at Moulay Ismail University, Morocco. He is member of IEEE. His research interests include information and communication technologies. A large part of his research projects are related to Error correction codes and Turbo codes. Idriss Chana published more than 20 papers in major journals and conferences in Information Theory.

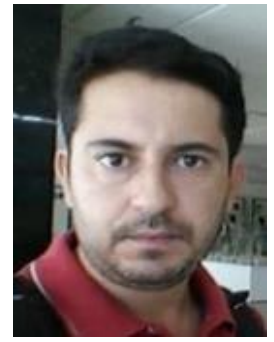

Youssef Hadi received his Ph.D. degree in Computer Sciences and Telecommunications from the Faculty of Sciences of the Mohammed V University, Rabat, Morocco in 2008. From 2009 to 2015, he was an Assistant Professor at Ibn Tofail University, Faculty of Sciences with LARIT laboratory. Currently he is an Associate Professor at the same university and he is the Vice Director of MISC Laboratory (Modeling of Information and Communication Systems). Also, he is a member of IEEE and he is Student Branch Member of Ibn Tofail University. Youssef Hadi research interests include Software Engineering, Model Driven Engineering, Cloud Computing and Signal Processing. 